mit zahlreichen empirischen Befunden belegt. Der Leser erhält übersichtliche Zusammenfassungen von Sachverhalten aus unterschiedlichen Quellen, wie z. B. die Kategorisierung von Kanalintervallen in unterschiedlichen Untersuchungsansätzen. Die Untersuchungsansätze werden hierbei nicht nur referiert, sondern auch zueinander in Beziehung gesetzt und bewertet.

Der zweite wesentliche Teil des Buches beschäftigt sich mit einer Sekundäranalyse von Daten aus dem AGF/GfK-Fernsehpanel, wobei ihm allerdings systematisch entgangen ist, dass es sich um das AGF/GfK-Fernsehpanel handelt und nicht um das Fernsehpanel der GfK.

Angesichts des geringen Umfangs an öffentlich zugänglicher Dokumentation ist es grundsätzlich schwierig, einen systematischen Überblick über das System der Fernsehzuschauerforschung in Deutschland zu geben. Insgesamt gelingt das zwar, in einigen Details aber - so z. B. bei der Beschreibung der sog. PIN-Daten als Übergang von der haushalts- zur personenbezogenen Messung des Fernsehverhaltens, von Instrumenten zur Qualitätskontrolle der Daten oder des Gewichtungsverfahrens - werden Sachverhalte nicht korrekt dargestellt.

Ausführlich und auch für den verständigen Leser transparent werden die Schritte beschrieben, mit denen der Datensatz für die Sekundäranalyse erstellt wird. Es wird hierbei schnell klar, dass der Umgang mit stark disaggregierten Daten aus dem AGF/GfK-Fernsehpanel bereits eine erhebliche Herausforderung darstellt, so dass die Beschränkung auf exemplarische Einzeltage aus dem Untersuchungszeitraum 1995 bis 2005 gut nachvollziehbar ist. Eine Extrapolation der Ergebnisse von insgesamt einem Untersuchungstag für jeweils zwei Jahre Fernsehalltag bleibt natürlich angreifbar. Der grundsätzliche Ansatz, „normale“ Tage nach eigener Programmkenntnis auszuwählen, ist natürlich die einzig sinnvolle Vorgehensweise. Auch die Beschränkung auf die 55 am meisten genutzten Sender ist richtig, auch wenn empirisch bekannt ist, dass kurzzeitige Sehvorgänge negativ mit dem Sehvolumen der Sender korrelieren und die Anzahl der Sender zwischen 1995 und 2005 stark gestiegen ist. Insgesamt gewährleistet das Vorgehen jedoch einigermaßen kontrollierte c.p.-Bedingungen, die für den Untersuchungszeitraum - soweit dies durchführbar war - konstant sind. Inwieweit die Generierung eines Datensatzes auf Basis der durchgehenden Masse des Panels von 1995 bis 2005 bei einer durchgehenden Masse von $4 \%$ der aktuellen Stichprobe zu interpretierbaren Ergebnissen führt, ist eher zweifelhaft. Dieser Datensatz wird glücklicherweise auch nur an einer Stelle der Analysen verwendet.

Ettenhuber entwickelt unter Verwendung der in der Literaturstudie gewonnenen Befunde Hypothesen zur Beschleunigung des Fernsehverhaltens und überprüft diese mit Hilfe der entsprechend aufbereiteten Datensätze. Hierbei wird in zahlreichen Detailanalysen deutlich, wie sich das Fernsehverhalten im Laufe von 10 Jahren beschleunigt hat und welche Ursachen hierfür verantwortlich sind. Die verwendeten Begriffe, wie beispielsweise „Evaluation, kürzere und längere Rezeption“, werden im $\mathrm{Da}$ tenmaterial operationalisiert und ermöglichen eine plastische und gut lesbare Ergebnisdarstellung. Die Aufbereitung ist ausführlich und berücksichtigt die relevanten Sachverhalte.

Zusammenfassend lässt sich feststellen, dass der Leser in der Publikation von Ettenhuber zur Beschleunigung des Fernsehverhaltens Informationen zur Entwicklung des Fernsehverhaltens erhält, die durchaus mit dem Prädikat „innovativ“ versehen werden können.

Bernhard Engel

\section{Friedrich Krotz}

\section{Mediatisierung}

Fallstudien zum Wandel von Kommunikation

Wiesbaden: VS Verlag für Sozialwissenschaften, 2007. $-333 \mathrm{~S}$.

\section{8-3-531-15073-4}

Stellen wir uns für einen Moment folgende Situation vor: Wir suchen auf der Plattform YouTube nach einem Video zu einem bestimmten Thema und sehen plötzlich uns selbst in einem der dortigen Angebote agieren, z. B. auf einer öffentlichen Veranstaltung oder beim Flanieren durch eine Einkaufspassage. Gegen diese Veröffentlichung kann man nur dann Einwände erheben, wenn man als Person eindeutig im Vordergrund steht und nicht Teil einer Menschenmenge ist. Das ist nicht neu, hat aber eine neue Dimension erlangt, die wieder einmal das Verhältnis von Privatheit und Öffentlichkeit 
betrifft. Man wird Teil eines Medienangebots, ohne es zu wissen. Der Ausruf „Das bin ja ich“ wäre dann eine spezifische Form des Identitätserlebnisses, das sich ohne Weiteres in die von Friedrich Krotz dargestellten Fallstudien zum Wandel von Kommunikation integrieren ließe. Dieser Kommunikationswandel beschreibt eben auch einen Wandel der Gesellschaft. Das Buch will somit die Rolle verschiedener Medien für soziale Veränderungen verdeutlichen.

Vorgelegt wird eine überarbeitete und erweiterte Version der im Jahr 2001 erschienenen Habilitationsschrift, in der, so der Verfasser, nicht alles neu ist, was man zu lesen bekommt (vgl. S. 21), aber zum Teil in kürzerer und prägnanterer Form argumentiert wird und auch bislang Unveröffentlichtes zu finden ist (vgl. S. 19). So fasst Kapitel 4 mehrere Analysen zu interaktiven Medien zusammen, die hier erstmals publiziert werden. Kapitel 1 dient einer Präzisierung des Konzepts „Mediatisierung“, Kapitel 2 skizziert mehrere theoretische Zugänge, die in Grundüberlegungen zu einer Systematik der Medienkommunikation einfließen (Kapitel 3). Kapitel 5 stellt die interpersonale mediatisierte Kommunikation (z. B. via Mobilfunk oder EMail) in den Vordergrund, Kapitel 6 diskutiert die Angemessenheit des Begriffs „Massenkommunikation" vor dem Hintergrund einer digitalen Medienvielfalt, Kapitel 7 kritisiert einen spezifischen Forschungsansatz („digitale Spaltung"), dem in Kapitel 8 eine knappe Schlussbemerkung folgt. Eine gewisse Heterogenität kann man dem Buch nicht absprechen, ebenso Redundanzen in der Argumentationsführung; aber das Rahmenthema bleibt im Fokus.

Mediatisierung lautet das Stichwort, das im Sinne des Autors einen gesellschaftlichen Metaprozess beschreibt. Damit soll gesagt werden, „dass es sich um lang andauernde und kulturübergreifende Veränderungen handelt, um Prozesse von Prozessen gewissermaßen, die die soziale und kulturelle Entwicklung der Menschheit langfristig beeinflussen [...].“ (S. 27) Friedrich Krotz zeigt, dass sich diese grundlegenden Veränderungen sowohl im Bereich der nicht-interaktiven als auch in verstärktem Maße in verschiedenen Formen interaktiver Kommunikation zu realisieren beginnen. Dass sich audiovisuelle Angebote an vielen Orten wahrnehmen lassen, ist nur ein Mosaikstein in dem Bild, das hier von einer von Medien durchdrungenen Alltagswelt gezeichnet wird. Diese Ausweitungen von Zugangsmöglichkeiten auf der einen und Formen der Erreichbarkeit auf der anderen Seite werden auch mit dem Begriff der Entgrenzung beschrieben (vgl. S. 96), wobei der Autor eine zeitliche, räumliche und sozial/situative Dimension unterscheidet. So ist es seltener der Fall, dass man an einem bestimmten Ort sein muss, um etwas wahrnehmen zu können. Dass sich immer häufiger Situationen benennen lassen, in denen sich die Menschen vor dem Bildschirm oder Medienangebot so verhalten wie die Menschen in dem Medienangebot, dürfte auch unbestritten sein. Diese Orientierungsfunktion muss dennoch nicht mit einer 1:1-Nachahmung gleichgesetzt werden. Der Kreis kann sich schließen, wenn Verhaltensweisen des Alltags für Medienangebote eine so hohe Attraktivität gewinnen, dass sie in diesen der Gesellschaft noch einmal vorgeführt werden.

Aber der eigentliche Fall der interaktiven Kommunikation, der auch im Sinne einer mediatisierten Kommunikation interpretiert werden kann, vollzieht sich insbesondere in einem Bereich, den Friedrich Krotz in Anlehnung an McMillan als „user-to-system-interactivity“ bezeichnet (S. 123). Computerspiele sind dafür ein gutes Beispiel, weil, so der Verfasser, „während des Spielprozesses ein wechselseitiger „Dialog“ statt[findet].“ (S. 123) Ein Nutzer und ein System, bestehend aus Hard- und Software, stellen sich hier immer wieder neu aufeinander ein. Insofern hat diese Mediennutzung eine andere Qualität: „Auch Fernsehrezeption ist ein Fall von Mensch-Maschine-Kommunikation, aber im Unterschied zum interaktiven Kommunizieren bezieht sich der Fernsehapparat nicht auf den Rezipienten und sein Verhalten vor dem Bildschirm, außer dass er gegebenenfalls unterschiedliche Programme anzeigt". (S. 124) Über den berühmten Turing-Test und das von Weizenbaum entwickelte Programm ELIZA spannt der Autor den Bogen bis hin $\mathrm{zu}$ modernen elektronisch gesteuerten Systemen, in denen sich diese Art der Kommunikation auf spielerische Art vollzieht, z. B. eine Aluminium-Plastikfigur wie Waldi, die einen vierbeinigen Hund simulieren soll. Der Autor referiert eine Untersuchung, wonach Vorschulkinder den „Artificial Dog“ mit den gleichen Wortkategorien behandeln wie einen Stoffhund, aber das Simulationsmodell mit integriertem Computer insgesamt mit größerer Vorsicht behandeln. Ebenso wird die Frage diskutiert, ob solche Simulationen für Zielgrup- 
pen, die einen „richtigen“ Hund nicht mehr halten können, ein Substitut darstellen könnten, z. B. für alte und kranke Menschen. Diese Frage löst verständlicherweise ethische Debatten aus, die vom Autor auch diskutiert und kommentiert werden. Entscheidend aber ist an diesen Beispielen, dass sie bereits mehr als einen Möglichkeitsraum aufzeigen. Eine Zunahme solcher und ähnlicher Entwicklungen fügt sich als weiterer Mosaikstein in ein Bild einer mehr und mehr mediatisierten Gesellschaft ein. Darum geht es Friedrich Krotz, wenn er an zahlreichen Fallstudien die Verschmelzung von Alltag und Medienrealität verdeutlicht.

Für diesen umfassenden Wandlungsprozess einen geeigneten theoretischen und methodischen Zugang zu benennen, ist eine große Herausforderung. Während die Fallstudien ohne Zweifel eine Nähe zu dieser Thematik vermitteln, sind die sozialwissenschaftlichen Grundlagentheorien (z. B der Symbolische Interaktionismus, die Cultural Studies und der „menschenkundliche“ integrative Ansatz von Norbert Elias [diese Formulierung wählt der Verfasser auf S. 18]) davon etwas entrückt. An der Verknüpfung der Mikro-(Fallstudien) und der Makroebene (Was bedeuten diese Entwicklungen für das gesellschaftliche Zusammenleben?) gilt es also noch intensiv zu arbeiten. Dem „unterschätzten Medium“ Computerspiele (S. 161) soll nach Krotz dabei wohl vermehrt Aufmerksamkeit zukommen.

Insgesamt wird also ein breites Forschungsprogramm skizziert, dessen Grenzen angesichts der sehr grundlegenden Bedeutung des Metaprozesses „Mediatisierung“ schwer zu ziehen sind. Hier ist noch eine Menge Ordnung in die Vielfalt zu bringen. Es ist eben ein Unterschied, ob man über die praktischen Konsequenzen des Mobiltelefons oder von Medienangeboten im öffentlichen Raum spricht, oder sehr grundsätzlich feststellt: „Identität, die Struktur des Menschen, seine Beziehung, seine alltäglichen Erfahrungen beruhen immer in erster Linie auf seiner Kommunikation und seinem Erleben dieser Kommunikation. Das geschieht auf der Ebene von Inhalten, aber vorgängig schon auf der Ebene der Kommunikationsstrukturen, in denen Menschen leben." (S. 210) Friedrich Krotz arbeitet an den Pfeilern, die zwischen diesen beiden Beschreibungsmodi eine Brücke tragen können.

Michael Jäckel

\section{Otfried Jarren / Patrick Donges (Hrsg.) \\ Ordnung durch Medienpolitik?}

Konstanz: UVK, 2007. - 411 S.

ISBN: 3-896-69585-1

Eigentlich handelt es sich hier um die Erträge eines Kolloquiums, das anlässlich des 75 . Geburtstages von Ulrich Saxer, langjähriger Ordinarius in Zürich, zu demselben Thema abgehalten wurde. Auch wenn ihm der Band gewidmet ist, ist er doch keine Denkschrift, und das ist auch gut so. Denn hier greift der Gefeierte kräftig in die Diskussion ein, gibt Fragestellungen vor und setzt sich mit vielen der Beiträge differenziert auseinander. Die Herausgeber sorgten zugleich für eine erkennbare Homogenität der Darstellungen, die dem Band einen weitaus größeren inneren Zusammenhang gibt, als es in den Mischangeboten üblicher Denkschriften der Fall ist. Er kann durchaus als repräsentativer Überblick zu ordnungspolitischen Ansätzen in der Medienpolitik-Forschung dienen.

Die Herausgeber stellen die Aufsatzsammlung unter das Postulat, „Politik soll soziale Ordnungen ermöglichen“, ebenso gilt, dass „Medien eine öffentliche Aufgabe wahrzunehmen haben“. Als gemeinsame Leitfrage wurde den Autoren vorgegeben, ob „'Ordnung durch Medienpolitik' in wissenschaftlich-theoretischer Perspektive wie aber auch politischpraktischer Perspektive je ein verfolgter Ansatz war“. (S. 9) Die Referenzländer liegen dabei im deutschsprachigen Raum Schweiz, Österreich und Deutschland, zu denen jeweils eine Analyse der nationalstaatlichen Ebene vorgelegt wird (Bonfadelli/Meier; Langenbucher; Vowe). Allerdings findet sich nur eine explizit komparative Studie, sie fragt nach den medienpolitischen Leitbildern in den drei kulturell verwandten, historisch aber teilweise sehr divergenten Staaten (Künzler/Schade).

Nationale Medienordnungen außerhalb des deutschsprachigen Raums spielen keine Rolle in dem Sammelband, allerdings beschäftigen sich Artikel mit der internationalen Ebene, wobei die Medienordnung der Europäischen Gemeinschaft thematisiert wird (Dörr), sowie der Einfluss der Weltorganisationen UNESCO und WTO auf nationale Medienregulierung (Puppis). Im weitaus umfangreichsten, dritten Teil steht die Perspektive der Wissenschaft im Mittelpunkt, genauer genommen geht es 